\title{
Inflammation in gout: mechanisms and therapeutic targets
}

\section{Authors:}

Alexander So

Fabio Martinon

1 Service of Rheumatology, CHUV and University of Lausanne, Lausanne, Switzerland

2 Department of Biochemistry, University of Lausanne, Epalinges, Switzerland.

Keywords: Gout, Interleukin-1 $\beta$, monosodium urate crystals, Inflammation

Competing interests:

AS: consultant for Novartis, AstraZeneca, Menarini. 


\section{Key points:}

- Inflammatory cytokines, in particular IL-1b are key mediators of gouty inflammation

- Multiple regulatory pathways modulate the activity of the inflammasome and the release of

IL1b

- Diet influences hyperuricemia as well as the inflammatory state of macrophages in gout

- The resolution of gouty inflammation is regulated

\section{Biographies:}

Alexander So is head of the Service of Rheumatology at the University Hospital of Lausanne and professor of rheumatology at the University of Lausanne. He obtained his medical training in Cambridge and London, and his $\mathrm{PhD}$ at the University of London. His research interest is the role of microcrystals in rheumatic diseases, particularly in gout and in osteoarthritis. His collaboration with Jürg Tschopp led to the use of IL-1 inhibition as a treatment of acute gout and he has participated in a number of clinical and experimental studies of IL1 inhibition in crystal diseases. Fabio Martinon received his Ph.D. from the University of Lausanne, Switzerland, for his work on the characterization of the Inflammasome in the laboratory of Jürg Tschopp. He trained as a postdoctoral fellow in the laboratory of Laurie H. Glimcher at the Harvard School of Public Health, in Boston. $\mathrm{He}$ is currently an Associate Professor in the Department of Biochemistry at the University of Lausanne. His laboratory is focused on the characterization of signalling pathways triggered by perturbations of cellular homeostasis and their role in inflammation, inflammatory diseases and cancer. 


\begin{abstract}
The acute symptoms of gout are triggered by the inflammatory response to MSU crystals, mediated principally by macrophages and neutrophils. Innate immune pathways are of key importance and in particular the activation of the NLRP3-inflammasome leading to release of IL-1 $\beta$ and other pro-inflammatory cytokines. This review will highlight recent advances in our understanding of both positive and negative regulatory pathways as well as the genetic and environmental factors that modulate the inflammatory response. Some of these pathways can be manipulated and open novel therapeutic opportunities for the treatment of the acute attack.
\end{abstract}




\section{Introduction}

Gout has become the most common cause of inflammatory arthritis, and its epidemiology worldwide points to an increase in incidence and prevalence in both developed and developing countries '. It is due to hyperuricemia (serum urate levels $>7 \mathrm{mg} / \mathrm{L}$ [420 umol/L] leading to formation and deposition of monosodium urate (MSU) crystals. Clinically, it is characterized by acute episodes of joint inflammation, usually affecting a single joint, interspersed by symptom free periods of variable duration. If untreated, it typically progresses to the formation of urate deposits (tophi) in soft tissues, recurrent attacks of arthritis affecting multiple joints and progressive joint destruction. Other complications include renal deposits of uric acid that can provoke renal failure and the formation of renal stones. These and other clinical features have been reviewed recently by Dalbeth ${ }^{2}$.

Gout is now regarded as a prototypical inflammatory disease driven by activation of the innate immune system and has also been termed an "autoinflammatory disease". However this classification is misleading, for unlike hereditary autoinflammatory disorders, the acute trigger of gout is MSU crystals. Uric acid itself is an endogenous and ubiquitous metabolite that is considered to possess little pro-inflammatory properties, and crystal formation is needed to provoke clinically observed inflammation.

The study of the underlying mechanisms of gouty inflammation has given us remarkable insights into the control of the inflammasome and pro-inflammatory cytokine release. Nevertheless, we must bear in mind some of the other distinguishing features of gout: 1) the attack is usually self-limiting, and 2) that crystals can be present without an inflammatory response. These observations imply that there are regulatory mechanisms that modify the acute inflammatory response, and a thorough understanding of pro- as will as anti-inflammatory pathways may help to develop new strategies for 
treatment. In this review we discuss the recent advances in the field of gout inflammation and new therapeutic strategies emerging to manage acute gout attacks.

\section{URIC ACID MEDIATED INFLAMMATION}

\section{Activation of the NRLP3 Inflammasome by monosodium urate crystals}

MSU crystals trigger an inflammatory response from macrophages. The crystals are first taken up by macrophages and promote the assembly and activation of the NLRP3 inflammasome ${ }^{4}$. Inflammasomes are cytosolic multiprotein complexes that can initiate inflammatory responses ${ }^{5}{ }^{6}$ Inflammasomes assemble when pattern-recognition receptors such as NLRP3 sense activating signals that reach the cytosol of the cell. This leads to the oligomerization of the pattern-recognition receptor and the recruitment to the complex of adaptor proteins and effector enzymes (Figure 1). NLRP3 inflammasomes are formed by the recruitment of the adaptor ASC and subsequent recruitment of caspase-1. Following initial oligomerization within the inflammasome, ASC can further auto-assemble into high molecular weight oligomers. This process, referred to as "prionlike" polymerization, amplifies the signals and virtually engages all ASC molecules into one active cellular complex '? Recruitment and oligomerization of caspase-1 by this structure leads to activation and proteolytic processing of its substrates.

The cytokines IL-1 $\beta$ and IL-18 are cleaved and activated by caspase-1. In gout, inflammasome mediated IL-1 $\beta$-release triggers an important inflammatory response, with vasodilatation and rapid recruitment of neutrophils to the site of crystal deposition, and thereby drives acute inflammatory episodes ${ }^{8}$.

Additional caspase-1 substrates such as Gasdermins are emerging as downstream effectors of inflammasome engagement ${ }^{\circ}$. Gasdermins promote cell death upon inflammatory caspases activation ${ }^{10 . "}$. Caspase-1 or caspase-11 cleave Gasdermin D (GSDMD) to release its N-terminal portion that then polymerizes at the plasma membrane forming cytotoxic pores. These pores alter cellular integrity and result in cells death by pyroptosis. Pyroptosis, also known as inflammatory 
caspases-mediated cell death, differs from apoptotic caspases-mediated cell death in that it result into the release of cytosolic content of cells, including a plethora of proinflammatory mediators and danger signals. Pyroptosis can therefore amplify the inflammatory response and facilitate the release of cytokines including IL-1 $1 \beta$. Whether this pathway contributes to inflammation in gout remains to be established.

In addition to inflammatory caspases, other proteases can contribute to IL-1 maturation ${ }^{12}$. In the absence of the inflammasome, neutrophils can process proIL-1 $\beta$ by the activity of neutrophilderived serine proteases such as proteinase-3 (PR3) elastase and cathepsin $G^{12,13}$. Other serine proteases can also process IL-1 ${ }^{{ }^{14}}$. Some metalloproteinases and granzyme A have also been proposed to trigger the proteolytic activation IL-1 $\beta^{15}{ }^{16}$. It is unclear whether these pathways function as an amplifier of the inflammatory reaction, for example in tissues with robust neutrophil recruitment, or function as backup mechanisms that maintain IL-1 $\beta$ production in condition where inflammasome proteins are absent or inhibited.

Despite the fact that activation of IL-1 $\beta$ production and NLRP3 inflammasome's role in gout is well described, the upstream pathway that links monosodium urate crystals to NLRP3 activation are poorly understood. We can dissect inflammasome engagement into two prerequisite steps: priming and activation. Relying on two signals is a key feature of most inflammasomes and increases the specificity of the response and avoids inappropriate firing of the pathway.

\section{MECHANISMS OF INFLAMMATION: PRIMING}

Priming, also known as signal $1^{17}$, controls the expression of all components required for the assembly and activation of the inflammasome and contribute to the expression of the precursor proteins that are substrate of inflammatory caspases. This inflammasome-competent stage is achieved as a result of an inflammatory milieu and can originate by the engagement of innate immune receptors such as Toll-like receptors (TLRs) or as part of an auto-amplification loop via IL$1 \beta$ itself. 
Signalling pathways mediated by cell surface receptors coordinates the innate immune response. The best known are the TLR family of receptors, and in particular, TLR2 and TLR4 have been implicated in gouty inflammation. Earlier work showed that TLR2 and TLR4 deficient mice have an impaired neutrophil response to MSU in the air pouch model ${ }^{18}$. A direct interaction between MSU crystals and the TLRs was postulated, as macrophages deficient for these receptors did not take up MSU crystals as efficiently as their wild type macrophages. Subsequent data suggest that TLRs regulate gouty inflammation by recognition of ligands that "prime" monocytes and macrophages to produce proIL-1 $\beta$. MRP 8 and 14 are endogenous ligands of TLR4 and are secreted upon activation of phagocytes. Patients with gout and mice that are injected with MSU produced high levels of MRP 8 and 14, and genetic deletion of MRP 14 reduced the response to MSU in mice ${ }^{19}$. Another ligand that may play a role in macrophage priming in gout is free fatty acids. In a murine model of gout, arthritis was only observed when mice were injected with both C18 free fatty acids (FFA) and MSU crystals, and injection of MSU alone or C18 FFA alone was not sufficient to elicit inflammation. Furthermore, it was demonstrated that TLR2 is the receptor that mediated C18 FFA's effects on macrophages ${ }^{20}$. The mechanisms of how TLRs can regulate inflammation have recently been reviewed ${ }^{21}$.

Other factors such as Granulocyte-macrophage colony-stimulating factor (GM-CSF) and the complement C5a have been proposed to affect priming in gout. In GM-CSF-neutralized mice, lower levels of IL-1 $\beta$ were observed following stimulation with MSU crystals. These monocytes also exhibited decreased expression of NLRP3 and proIL-1 ${ }^{23}$. Similarly, treatment with C5a increased the expression of IL-1 $\beta$ and IL-18 and exacerbated MSU-mediated peritonitis in a mouse model of gout $\{$ An, 2014 \#69; Khameneh, 2017 \#68\}.

While priming is necessary for inflammasome assembly, this step is non-specific and can result from various conditions and signals that promote an underlining inflammatory response. It provides 
an environment for inflammasome engagement, but it is not sufficient to trigger the inflammasome pathway per se.

\section{MECHANISMS OF INFLAMMATION: CATALYZING INFLAMMASOME ASSEMBLY}

A second signal (signal 2) is required for inflammasome activation. This signal is more specific than signal 1. It directly drives post-transcriptional and translational aggregation and polymerization of the inflammasome components. The mechanisms by which monosodium urate crystals trigger signal 2 to promote NLRP3 activation are still poorly understood. However several steps commonly found upstream of NLRP3 are involved.

Perturbation of cellular ionic balances, in particular potassium efflux and calcium influx, is the first feature characteristic of NLRP3 inducers ${ }^{24,25}$. This ionic perturbation is necessary for mitochondrial reactive oxygen (ROS) generation upstream of NLRP3 inflammasome assembly. ROS production is also an essential step required for inflammasome formation, it is increased by MSU-mediated Leukotriene B4 \{Amaral, 2012 \#22\} and may contribute to engage NEK7, a member of the family of mammalian NIMA-related kinases (NEK proteins). NEK7 directly binds NLRP3 and may be the common NLRP3 activating ligand ${ }^{2628}$. The important questions that remain to be resolved are how NEK7 interacts with NLRP3 and the mechanisms by which the crystals promote the ionic changes that ultimately engage the NLRP3 activating cascade.

\section{IL-1 $\beta$ IS A KEY CYTOKINE IN GOUT}

IL-1 $\beta$ is a cytokine that acts on multiple cell types to elicit inflammatory responses ${ }^{29}$. It promotes vasodilatation leading to the recruitment of monocytes and neutrophils to sites of tissue insults, a response that is crucial in combating infection and restoring tissue homeostasis. However sustained IL-1 $\beta$ secretion can result in the production of matrix degrading enzymes that breaks down cartilage and bone ${ }^{30}$. At the systemic level, IL-1 elicits a fever response by acting directly on the hypothalamic temperature regulation centre ${ }^{31}$. 
IL-1 $\beta$ is mainly produced by innate immune cells and signals to target cells by binding to the IL1 receptor type 1 (IL1R1). Once activated, IL1R1 and its co-receptor IL1 receptor accessory protein (IL1RAP) recruit a signalling complex that shares components with TLR signalling, leading to the activation of pro-inflammatory transcription factors including nuclear factor $\kappa \mathrm{B}(\mathrm{NF} \kappa \mathrm{B})$, as well as p38, c-Jun N-terminal kinase (JNK) (Figure 2). These transcription factors in turn promote the transcriptional upregulation of chemokines and proinflammatory mediators that orchestrate the IL1-mediated inflammatory response.

It is now widely accepted that IL- $1 \beta$ is a pivotal cytokine in acute gout ${ }^{32}$, but a role for IL- $1 \alpha$ cannot be ruled out. IL-1 $\alpha$ is released during crystal-induced inflammation and mice with deletion of the $I L-1 B$ gene are still capable of mounting a neutrophil response ${ }^{33}$. The clinical relevance of IL-1 is supported by data from a number of different clinical studies of IL-1 inhibition ${ }^{34}$. Although IL-1 inhibition is not recommended as a first-line anti-inflammatory treatment, the results from clinical trials (of canakinumab) and cohort studies of patients who have received anakinra as treatment of acute flares showed a rapid onset of pain relief and it was observed that nearly all treated patients responded. Patients who had subsequent flares responded equally well when treated with again with canakinumab ${ }^{35}$ and in our personal experience, anakinra was also effective when given for recurrent flares. The clinical experience with different IL-1 inhibitors in the treatment of gout is detailed in a later section.

\section{Other cytokines contributing to inflammation in Gout}

IL-8, also known as CXCL8 is a macrophage-secreted chemokine that acts principally on neutrophils. Recent data showed that it is significantly increased during an acute attack in three different cohorts of patients, and interesting, its levels remained high during the intercritical phase of gout and also in patients that had concomitant diabetes. In contrast, other co-morbidities that are commonly seen in gout (such as cardiovascular disease and chronic kidney disease) were not associated with high IL-8 levels ${ }^{36}$. The mechanisms underlying these observations have not yet been 
elucidated, but suggest that neutrophil recruitment and neutrophil activation are key inflammatory pathways.

\section{Soluble uric acid as a modulator of inflammation}

In gout, it is the formation of MSU crystals that trigger acute inflammation, but data show that hyperuricemia can modulate the inflammatory response. Hyperuricemia by itself, in the absence of crystals, is able to skew the leukocyte response towards a more inflammatory pattern through epigenetic modifications of histone methylation. In patients with hyperuricemia, this was shown by enhanced production of IL-1 $\beta$ and IL-6 and concomitant reduction of IL1RA release ${ }^{39}$.

\section{INFLUENCE OF DIETARY FACTORS AND THE MICROBIOME ON GOUT INFLAMMATION}

Clinical observations indicate that dietary factors play a major role in gout, and the link between patterns of food consumption and hyperuricemia and gout has long been established ${ }^{40}$. More recently, the role of particular foods in triggering an acute attack has been raised, and findings from an internet-based survey suggest that high purine content increases the risk of an acute attack of gout five-fold ${ }^{4}$. However, the components of food and how they can lead to an attack remains to be identified. As mentioned earlier, long chain (C18) fatty acids may play a role in priming macrophages to release IL- $1 \beta$ when they phagocytose MSU crystals ${ }^{20}$.

Another mechanism that has been studied recently is the gut microbiome and its interaction with inflammatory cells and how this affects inflammation. Using a mouse model of gout, it was shown that germ-free mice showed attenuated MSU-induced inflammation and this effect was reproduced by antibiotic treatment. They went on to show that these effects were mediated by acetate, a shortchain fatty acid that is released by gut bacteria. Acetate acts via the macrophage GPR-43 receptor to modulate inflammasome activation and IL-1 $\beta$ production. Restoring the normal gut flora in germ 
free mice, and the addition of acetate restored the inflammatory properties ${ }^{42}$. The same group further went on to show that a high fibre diet can attenuate arthritis in the same murine model of gout ${ }^{43}$. In that study it was proposed that increased production of acetate and other short chain fatty acids resulting from the high fibre diet, regulated resolution of inflammation possibly by impacting neutrophils ${ }^{43}$. These studies suggest that environmental factors such as microbial metabolites can differentially regulate the cell types and pathways of gouty inflammation. The changes in the microbiome in gout patients have not been extensively studied. There is one report that found that gout patients had a different bacterial flora compared with control subjects and was similar to that found in diabetics ${ }^{4}$. These findings need to be reproduced in larger cohorts before we can draw clear conclusions on how the microbiome modulates inflammation in gout.

Although there is great interest in using diet to modulate hyperuricemia, its overall effect is modest. However, dietary factors may influence gouty inflammation. Recent clinical data suggest that higher dietary consumption of omega-3 fatty acids is associated with a lower frequency of acute gout flares ${ }^{45}$. Experimentally, $\quad-3$ fatty acids (eicosapentaenoic acid and docosahexaenoic acid) can inhibit NLRP3 inflammasome activation via a pathway that involves the G-protein coupled receptors GPR120 and GPR40 and $\beta$-arrestin2 ${ }^{46}$. These findings require confirmation by intervention trials using omega-3 in gout.

\section{GENETICS OF GOUTY INFLAMMATION}

Genetic studies including genome-wide association studies (GWAS) have identified dozen susceptibility loci associated with hyperuricemia and gout ${ }^{47}$. These loci mostly influence uric acid levels by affecting pathways such as renal and gut excretion of uric acid. While hyperuricemia may influence oxidative stress and thereby have some impact on inflammatory pathways, whether these polymorphisms may directly modulate inflammatory responses, beyond promoting uric acid crystallization, remain to be demonstrated. 
Two studies demonstrated an association of gout with functional variants in CARD8 ${ }^{48,49}$. CARD8 is a potential negative regulator of the NLRP3 inflammasome. It therefore possible that these polymorphisms may increase inflammasome activity and thereby contribute to the intensity or duration of NLRP3 engagement in gouty episodes. In addition there is genetic evidence for a role of TLR4 in gout. Employing a candidate gene approach, two studies, one performed in Han Chinese and the other in patients with a European ancestry, found a significant association between the same genetic polymorphism of the TLR4 gene (rs2149356) with gout ${ }^{{ }^{\circ 0}, 51}$. These polymorphisms may impact the priming phase of inflammasome engagement or may have a broader impact on the inflammatory responses in these patients.

One study linked gout incidence with a polymorphism within the gene Peroxisome proliferatoractivated receptor gamma coactivator 1- $\beta(P P A R G C 1 B)\{$ Chang, 2017 \#70\}. It was shown that this variant increased NLRP3 and IL-1 $\beta$ expression. Because PPARGC1B function as a regulator of PPAR $\gamma$, a master regulator of metabolism, this genetic evidence may link metabolic deregulation with gouty inflammation.

\section{FACTORS CONTRIBUTING TO THE RESOLUTION OF INFLAMMATION}

Monocytes and macrophages are the major cellular sources of IL-1, but at the site of inflammation, neutrophils predominate. The major pro-inflammatory role of the neutrophil and the mechanisms of interaction between MSU crystals and the neutrophil has been reviewed ${ }^{52}$. Interestingly, neutrophils also probably play a major role in the resolution of acute gout, by the formation of neutrophil extracellular traps (NET). This process is favoured by high neutrophil concentrations in the experimental setting $\left(>10 \times 10^{6} / \mathrm{ml}\right)$, and results in the formation of cellular aggregates that contain cellular debris, DNA as well as neutrophil proteases released into the NETs ${ }^{53}$. NET formation is dependent on the generation of ROS and recent evidence also implicates molecules that regulate necroptosis via the RIPK3 pathway ${ }^{54}$. In the absence of RIPK3, NET formation was inhibited 
completely. Once formed, cellular aggregates containing NETs can degrade a wide range of inflammatory cytokines rapidly and in experimental models, inhibition of NET formation results in more severe and persistent gouty inflammation, whereas when NET formation is not impaired, there is a spontaneous resolution of joint inflammation after day $3^{53}$. These results show that neutrophils have a dual role in gouty inflammation: in the initial phase when inflammation is amplified by recruited neutrophils, as well as the resolution of inflammation (Figure 3). Currently, we do not have any therapies that can modulate this process.

Anti-inflammatory cytokines also contribute to the resolution of the acute inflammatory process. In animal models, addition of exogenous TGF $\beta 1$ reduced experimental inflammation ${ }^{37}$; in man, TGF $\beta 1$, IL-10 and IL1RA were elevated in synovial fluid and are associated with spontaneous resolution of gouty arthritis $\{$ Chen, $2011 \# 38\}$. Other factors may contribute to timely resolution of inflammation in gout. For example, the protein Annexin A1, a potential inhibitor of phospholipase A2 has been shown to decrease inflammation and promote resolution in mouse models of gout \{Galvao, 2017 \#67\}.

\section{THERAPEUTIC STRATEGIES TARGETING INFLAMMATION PATHWAYS IN GOUT}

Treating gout requires two complementary approaches, one aimed at lowering uric acid level and the other to reduce inflammation. NSAIDs, colchicine and corticosteroids are commonly used and are effective in relieving pain and inflammation of the acute attack, however our recent insights into the biology of inflammation open the way to new therapeutic strategies (Figure 3).

\section{Modulators and inhibitors of the NLRP3 inflammasome}

As the NLRP3 inflammasome is a key pathway in the sensing of MSU crystals, strategies that impede its activation or affect its activity could reduce gouty inflammation. Interestingly, colchicine 
blocks MSU crystals mediated NLRP3 activity in macrophages ${ }^{32}$ probably by inhibiting microtubule-driven rearrangement of mitochondria following NLRP3 engagement with crystals ss.

Other molecules that target key steps leading to NLRP3 assembly have been described to impact inflammation. The ketone bodies $\beta$-hydroxybutyrate suppresses inflammasome activation in response to monosodium urate crystals ${ }^{56}$. These ketone bodies are produced in the liver of mammals during nutrient deprivation. Hence, starvation attenuated caspase- 1 activation and IL-1 $\beta$ secretion in mouse models caloric restriction ${ }^{56}$. Similarly, a ketogenic diet protected rats from urate crystals mediated gouty flares ${ }^{57}$. Mechanistically, $\beta$-hydroxybutyrate has been proposed to inhibit potassium efflux upstream of NLRP3 and to directly impact inflammasome assembly ${ }^{56}$. However a possible effect on priming has also been suggested ${ }^{57}$.

Multiple studies in gout have also shown beneficial effects of compounds that inhibit ROS production and decrease oxidative stress. Epigallocatechin gallate, a potent antioxidant polyphenol found in green tea has been shown to inhibit neutrophil infiltration and IL-1 $\beta$ secretion in a mouse model of monosodium urate crystal-mediated peritonitis ${ }^{s}$. Morin, a natural flavonol, was found to impair monosodium urate crystal-induced inflammation in mouse macrophages ${ }^{9}$. Rebamipide, a gastroprotective drug was shown to suppresses monosodium urate crystal-mediated Interleukin-1 $\beta$ activation and release in human THP-1 cells ${ }^{60}$. Further studies are required to interrogate possible repositioning of this drug in gout. Xanthine oxidase inhibitors used in patients to decrease urate levels have also been shown to impact directly mitochondrial ROS production thereby inhibiting urate crystal mediated inflammasome activation ${ }^{6}$.

Inhibiting ROS production or potassium efflux are rather non-specific strategies that may have undesirable effects. Identification of more specific NLRP3 inhibitors could therefore present as more suitable therapeutics in gout. An example would be MCC950 (also known as CP-456,773 or CRID3) that has emerged as a potential drug of interest recently. This drug is a diarylsulfonylureacontaining compound that was initially identified as an inhibitor of extracellular ATP-mediated 
maturation of IL-1 ${ }^{\circ}$. This discovery preceded the initial description of the inflammasome and the identification of NLRP3 as the main sensor of extracellular ATP signals. More recently it was demonstrated that MCC950 specifically inhibit the NLRP3 inflammasome ${ }^{63}$. It blocks NLRP3induced ASC oligomerization in mouse and human macrophages without affecting the activation of NLRP1, AIM2, or NLRC4 inflammasomes. Several NLRP3 activators, including monosodium urate crystals where inhibited by MCC950 ${ }^{63,64}$, indicating that it may directly act on a conserved NLRP3 activating mechanism, However, exactly how this drug affects NLRP3 activation is not yet clear.

\section{Inhibitors of IL-1 $\beta$ maturation}

Considerable effort has been taken to develop specific caspase-1 inhibitors. VX-765, an orally available pro-drug, is the best studied. This drug is rapidly hydrolyzed by plasma and liver esterases into a potent and selective inhibitor of caspase- ${ }^{\circ 5}$. In animals, VX765 ameliorated the severity and progression of disease in a mouse model of collagen-induced arthritis ${ }^{6}$. Caspase- 1 inhibitors have also been shown to decrease IL-1 $\beta$ production and cartilage damage in a model of chronic destructive joint inflammation ${ }^{67}$. However, its effects in gout have not been explored. Of particular importance will be the specificity of the inhibitor for caspase-1, as caspases share a very conserved catalytic core and off-target inhibition of apoptotic caspases may cause undesirable consequences.

Based on studies that showed a role for serine proteases in proIL-1 $\beta$ inhibitors of serine proteases could be of interest, either as monotherapy or in combination with caspase-1 inhibitors in gout. Alpha-1-anti-trypsin (AAT) is a member of the serpin superfamily that inhibits many serine proteases. Recombinant human AAT-Fc fusion protein was found to be very effective in a mouse model of gouty arthritis ${ }^{68}$. AAT can modulate inflammation at multiple levels and it is still unclear whether the effects observed in the gout model is directly caused by the inhibition of IL-1 $\beta$ processing. Yet theses data indicate that AAT-fc and possibly other serine protease inhibitors could be of therapeutics of interest for gout attacks. 


\section{IL-1 inhibitors}

The evidence for the clinical efficacy of IL-1 inhibition in acute gout has been recently reviewed ${ }^{34}$. Two molecules are currently available, but only canakinumab has an indication for acute gout in the EU. The mechanism of action of the two drugs is different; canakinumab is a specific inhibitor of IL-1 $\beta$, and anakinra inhibits both IL- $1 \alpha$ and IL-1 $\beta$ binding to the IL1R 1 receptor. Their properties are listed in table 1.

IL-1 inhibition can relieve the acute symptoms of gout in patients who have not responded to conventional treatments or in whom the use of NSAIDs, colchicine or steroids are contraindicated. Its use in patients with severe renal and cardiac impairment, a clinical situation that commonly makes the choice of acute therapy difficult, has not been formally assessed in clinical trials, but in case series, no severe side effects have been reported ${ }^{34}$. In the clinical trials involving canakinumab, a significant reduction of gout flares was seen for for up to 6 months, however the drug is not registered for this indication. As drugs have not been tested in large number of patients, their use is restricted to patients who have "difficult-to treat" disease and their safety in terms of infectious complications needs to be considered when they are prescribed.

\section{Future considerations}

Recent years have shown considerable progress in the understanding of the mechanisms of inflammation and the role of innate immune sensors in gout, however several question remain unanswered. Three questions are of particular interest. First the detailed specific mechanism by which NLRP3 is activated upon exposure to monosodium uric acid crystal is still unclear. Among the possibilities emerging is that NLRP3 may act as a guardian of cellular integrity that detects perturbations triggered when innate immune cells attempt at engulfing large particulates ${ }^{69}$. This 
would imply that the size and possibly the chemical nature and shape of those crystals may affects immune responses. Second, the caspase-1 independent mechanisms of IL-1 production, the proteases engaged and what trigger their activation are still poorly understood. In this context it would be important to understand at what stage of the response these pathways contribute to the inflammatory phenotype and what are the cell types orchestrating this inflammasome-independent response. Finally, a key area of interest involves the mechanisms that initiate the gouty attacks, in patients that have persistent monosodium urate crystals deposits. It is still unclear whether specific initiation mechanisms contribute to triggering the inflammatory reaction, possibly by acting on priming signalling, or whether decrease in negative regulation of NLRP3 engagement are what promotes the inflammatory cascade. A better understanding of these questions may identify potential specific therapeutic strategies that will make the prevention and the management of gout more effective and specific. The study of this old disease provided us with a greater understanding of inflammatory pathways, solving the remaining questions still bears enormous potential for new discoveries of pathways and treatments that may impact several inflammatory diseases.

\section{References}

1. Kuo, C.F., Grainge, M.J., Zhang, W. \& Doherty, M. Global epidemiology of gout: prevalence, incidence and risk factors. Nat Rev Rheumatol 11, 649-62 (2015).

2. $\quad$ Dalbeth, N., Merriman, T.R. \& Stamp, L.K. Gout. Lancet (2016).

3. Neogi, T. et al. 2015 Gout classification criteria: an American College of Rheumatology/European League Against Rheumatism collaborative initiative. Ann Rheum Dis 74, 1789-98 (2015).

4. Martinon, F. \& Glimcher, L.H. Gout: new insights into an old disease. J Clin Invest 116, 2073-5 (2006).

5. Broz, P. \& Dixit, V.M. Inflammasomes: mechanism of assembly, regulation and signalling. Nat Rev Immunol 16, 407-20 (2016).

6. Martinon, F., Burns, K. \& Tschopp, J. The inflammasome: a molecular platform triggering activation of inflammatory caspases and processing of proIL-beta. Mol Cell 10, 417-26 (2002). 
7. Cai, X. et al. Prion-like polymerization underlies signal transduction in antiviral immune defense and inflammasome activation. Cell 156, 1207-22 (2014).

8. Chen, C.J. et al. MyD88-dependent IL-1 receptor signaling is essential for gouty inflammation stimulated by monosodium urate crystals. J Clin Invest 116, 2262-71 (2006).

9. Aglietti, R.A. \& Dueber, E.C. Recent Insights into the Molecular Mechanisms Underlying Pyroptosis and Gasdermin Family Functions. Trends Immunol (2017).

10. Abhishek, A. et al. In vivo detection of monosodium urate crystal deposits by Raman spectroscopy-a pilot study. Rheumatology (Oxford) 55, 379-80 (2016).

11. Kayagaki, N. et al. Caspase-11 cleaves gasdermin D for non-canonical inflammasome signalling. Nature 526, 666-71 (2015).

12. Netea, M.G., van de Veerdonk, F.L., van der Meer, J.W., Dinarello, C.A. \& Joosten, L.A. Inflammasome-independent regulation of IL-1-family cytokines. Annu Rev Immunol 33, 4977 (2015).

13. Sugawara, S. et al. Neutrophil proteinase 3-mediated induction of bioactive IL-18 secretion by human oral epithelial cells. J Immunol 167, 6568-75 (2001).

14. Mizutani, H., Schechter, N., Lazarus, G., Black, R.A. \& Kupper, T.S. Rapid and specific conversion of precursor interleukin 1 beta (IL-1 beta) to an active IL-1 species by human mast cell chymase. J Exp Med 174, 821-5 (1991).

15. Echtermeyer, F. et al. Syndecan-4 regulates ADAMTS-5 activation and cartilage breakdown in osteoarthritis. Nat Med 15, 1072-6 (2009).

16. Irmler, M. et al. Granzyme A is an interleukin 1 beta-converting enzyme. $J$ Exp Med 181, 1917-22 (1995).

17. Burns, K., Martinon, F. \& Tschopp, J. New insights into the mechanism of IL-1beta maturation. Curr Opin Immunol 15, 26-30 (2003).

18. Liu-Bryan, R., Scott, P., Sydlaske, A., Rose, D.M. \& Terkeltaub, R. Innate immunity conferred by Toll-like receptors 2 and 4 and myeloid differentiation factor 88 expression is pivotal to monosodium urate monohydrate crystal-induced inflammation. Arthritis Rheum 52, 2936-46 (2005).

19. Holzinger, D. et al. Myeloid-related proteins 8 and 14 contribute to monosodium urate monohydrate crystal-induced inflammation in gout. Arthritis Rheumatol 66, 1327-39 (2014).

20. Joosten, L.A. et al. Engagement of fatty acids with Toll-like receptor 2 drives interleukin1beta production via the ASC/caspase 1 pathway in monosodium urate monohydrate crystalinduced gouty arthritis. Arthritis Rheum 62, 3237-48 (2010).

21. Joosten, L.A., Abdollahi-Roodsaz, S., Dinarello, C.A., O'Neill, L. \& Netea, M.G. Toll-like receptors and chronic inflammation in rheumatic diseases: new developments. Nat Rev Rheumatol (2016).

22. Amaral, F.A. et al. NLRP3 inflammasome-mediated neutrophil recruitment and hypernociception depend on leukotriene B(4) in a murine model of gout. Arthritis Rheum 64, 474-84 (2012).

23. Shaw, O.M., Pool, B., Dalbeth, N. \& Harper, J.L. The effect of diet-induced obesity on the inflammatory phenotype of non-adipose-resident macrophages in an in vivo model of gout. Rheumatology (Oxford) 53, 1901-5 (2014). 
24. Petrilli, V. et al. Activation of the NALP3 inflammasome is triggered by low intracellular potassium concentration. Cell Death Differ 14, 1583-9 (2007).

25. Yaron, J.R. et al. $\mathrm{K}(+)$ regulates $\mathrm{Ca}(2+)$ to drive inflammasome signaling: dynamic visualization of ion flux in live cells. Cell Death Dis 6, e1954 (2015).

26. He, Y., Zeng, M.Y., Yang, D., Motro, B. \& Nunez, G. NEK7 is an essential mediator of NLRP3 activation downstream of potassium efflux. Nature 530, 354-7 (2016).

27. Schmid-Burgk, J.L. et al. A Genome-wide CRISPR (Clustered Regularly Interspaced Short Palindromic Repeats) Screen Identifies NEK7 as an Essential Component of NLRP3 Inflammasome Activation. J Biol Chem 291, 103-9 (2016).

28. Shi, H. et al. NLRP3 activation and mitosis are mutually exclusive events coordinated by NEK7, a new inflammasome component. Nat Immunol 17, 250-8 (2016).

29. Dinarello, C.A. Immunological and inflammatory functions of the interleukin-1 family. Annu Rev Immunol 27, 519-50 (2009).

30. Schlesinger, N. \& Thiele, R.G. The pathogenesis of bone erosions in gouty arthritis. Ann Rheum Dis 69, 1907-12 (2010).

31. Dinarello, C.A. Infection, fever, and exogenous and endogenous pyrogens: some concepts have changed. J Endotoxin Res 10, 201-22 (2004).

32. Martinon, F., Petrilli, V., Mayor, A., Tardivel, A. \& Tschopp, J. Gout-associated uric acid crystals activate the NALP3 inflammasome. Nature 440, 237-41 (2006).

33. Pazar, B. et al. Basic calcium phosphate crystals induce monocyte/macrophage IL-1beta secretion through the NLRP3 inflammasome in vitro. J Immunol 186, 2495-502 (2011).

34. Dumusc, A. \& So, A. Interleukin-1 as a therapeutic target in gout. Curr Opin Rheumatol 27, 156-63 (2015).

35. Alten, R. et al. Efficacy of Canakinumab on Re-Treatment in Gouty Arthritis Patients with Limited Treatment Options: 24-Week Results From $\beta$-RELIEVED and $\beta$-RELIEVED-II Studies. Arthritis Rheum 63, S402 (2011).

36. Kienhorst, L.B. et al. Gout Is a Chronic Inflammatory Disease in Which High Levels of Interleukin-8 (CXCL8), Myeloid-Related Protein 8/Myeloid-Related Protein 14 Complex, and an Altered Proteome Are Associated With Diabetes Mellitus and Cardiovascular Disease. Arthritis Rheumatol 67, 3303-13 (2015).

37. Liote, F. et al. Inhibition and prevention of monosodium urate monohydrate crystal-induced acute inflammation in vivo by transforming growth factor beta1. Arthritis Rheum 39, 1192-8 (1996).

38. Chen, Y.H. et al. Spontaneous resolution of acute gouty arthritis is associated with rapid induction of the anti-inflammatory factors TGFbeta1, IL-10 and soluble TNF receptors and the intracellular cytokine negative regulators CIS and SOCS3. Ann Rheum Dis 70, 1655-63 (2011).

39. Crisan, T.O. et al. Soluble uric acid primes TLR-induced proinflammatory cytokine production by human primary cells via inhibition of IL-1Ra. Ann Rheum Dis 75, 755-62 (2016).

40. Choi, H.K., Atkinson, K., Karlson, E.W., Willett, W. \& Curhan, G. Purine-rich foods, dairy and protein intake, and the risk of gout in men. N Engl J Med 350, 1093-103 (2004). 
41. Zhang, Y. et al. Purine-rich foods intake and recurrent gout attacks. Ann Rheum Dis 71, 1448-53 (2012).

42. Vieira, A.T. et al. A Role for Gut Microbiota and the Metabolite-Sensing Receptor GPR43 in a Murine Model of Gout. Arthritis Rheumatol 67, 1646-56 (2015).

43. Vieira, A.T. et al. Dietary fiber and the short-chain fatty acid acetate promote resolution of neutrophilic inflammation in a model of gout in mice. J Leukoc Biol 101, 275-284 (2017).

44. Guo, Z. et al. Intestinal Microbiota Distinguish Gout Patients from Healthy Humans. Sci Rep 6, 20602 (2016).

45. Abhishek, A., Valdes, A.M. \& Doherty, M. Low omega-3 fatty acid levels associate with frequent gout attacks: a case control study. Ann Rheum Dis 75, 784-5 (2016).

46. Yan, Y. et al. Omega-3 fatty acids prevent inflammation and metabolic disorder through inhibition of NLRP3 inflammasome activation. Immunity 38, 1154-63 (2013).

47. Merriman, T.R. An update on the genetic architecture of hyperuricemia and gout. Arthritis Res Ther 17, 98 (2015).

48. McKinney, C. et al. Multiplicative interaction of functional inflammasome genetic variants in determining the risk of gout. Arthritis Res Ther 17, 288 (2015).

49. Lee, Y.H. \& Bae, S.C. Association between functional NLRP3 polymorphisms and susceptibility to autoimmune and inflammatory diseases: a meta-analysis. Lupus (2016).

50. Qing, Y.F. et al. Association of TLR4 Gene rs2149356 polymorphism with primary gouty arthritis in a case-control study. PLoS One 8, e64845 (2013).

51. Rasheed, H. et al. The Toll-Like Receptor 4 (TLR4) Variant rs2149356 and Risk of Gout in European and Polynesian Sample Sets. PLoS One 11, e0147939 (2016).

52. Popa-Nita, O. \& Naccache, P.H. Crystal-induced neutrophil activation. Immunol Cell Biol 88, 32-40 (2010).

53. Schauer, C. et al. Aggregated neutrophil extracellular traps limit inflammation by degrading cytokines and chemokines. Nat Med 20, 511-7 (2014).

54. Desai, J. et al. PMA and crystal-induced neutrophil extracellular trap formation involves RIPK1-RIPK3-MLKL signaling. Eur J Immunol 46, 223-9 (2016).

55. Misawa, T. et al. Microtubule-driven spatial arrangement of mitochondria promotes activation of the NLRP3 inflammasome. Nat Immunol 14, 454-60 (2013).

56. Youm, Y.H. et al. The ketone metabolite beta-hydroxybutyrate blocks NLRP3 inflammasome-mediated inflammatory disease. Nat Med 21, 263-9 (2015).

57. Goldberg, E.L. et al. beta-Hydroxybutyrate Deactivates Neutrophil NLRP3 Inflammasome to Relieve Gout Flares. Cell Rep 18, 2077-2087 (2017).

58. Jhang, J.J., Lu, C.C. \& Yen, G.C. Epigallocatechin gallate inhibits urate crystals-induced peritoneal inflammation in C57BL/6 mice. Mol Nutr Food Res 60, 2297-2303 (2016).

59. Dhanasekar, C., Kalaiselvan, S. \& Rasool, M. Morin, a Bioflavonoid Suppresses Monosodium Urate Crystal-Induced Inflammatory Immune Response in RAW 264.7 Macrophages through the Inhibition of Inflammatory Mediators, Intracellular ROS Levels and NF-kappaB Activation. PLoS One 10, e0145093 (2015). 
60. Kim, S.K., Choe, J.Y. \& Park, K.Y. Rebamipide Suppresses Monosodium Urate CrystalInduced Interleukin-1beta Production Through Regulation of Oxidative Stress and Caspase1 in THP-1 Cells. Inflammation 39, 473-82 (2016).

61. Ives, A. et al. Xanthine oxidoreductase regulates macrophage IL1beta secretion upon NLRP3 inflammasome activation. Nat Commun 6, 6555 (2015).

62. Perregaux, D.G. et al. Identification and characterization of a novel class of interleukin-1 post-translational processing inhibitors. J Pharmacol Exp Ther 299, 187-97 (2001).

63. Coll, R.C. et al. A small-molecule inhibitor of the NLRP3 inflammasome for the treatment of inflammatory diseases. Nat Med 21, 248-55 (2015).

64. Primiano, M.J. et al. Efficacy and Pharmacology of the NLRP3 Inflammasome Inhibitor CP-456,773 (CRID3) in Murine Models of Dermal and Pulmonary Inflammation. $J$ Immunol 197, 2421-33 (2016).

65. Wannamaker, W. et al. (S)-1-((S)-2-\{[1-(4-amino-3-chloro-phenyl)-methanoyl]-amino\}-3,3dimethyl-butanoy 1)-pyrrolidine-2-carboxylic acid ((2R,3S)-2-ethoxy-5-oxo-tetrahydrofuran-3-yl)-amide (VX-765), an orally available selective interleukin (IL)-converting enzyme/caspase-1 inhibitor, exhibits potent anti-inflammatory activities by inhibiting the release of IL-1beta and IL-18. J Pharmacol Exp Ther 321, 509-16 (2007).

66. Zhang, Y. \& Zheng, Y. Effects and mechanisms of potent caspase-1 inhibitor VX765 treatment on collagen-induced arthritis in mice. Clin Exp Rheumatol 34, 111-8 (2016).

67. Joosten, L.A. et al. Inflammatory arthritis in caspase 1 gene-deficient mice: Contribution of proteinase 3 to caspase 1 -independent production of bioactive interleukin-1beta. Arthritis Rheum 60, 3651-62 (2009).

68. Joosten, L.A. et al. Alpha-1-anti-trypsin-Fc fusion protein ameliorates gouty arthritis by reducing release and extracellular processing of IL-1beta and by the induction of endogenous IL-1Ra. Ann Rheum Dis 75, 1219-27 (2016).

69. Hornung, V. et al. Silica crystals and aluminum salts activate the NALP3 inflammasome through phagosomal destabilization. Nat Immunol 9, 847-56 (2008). 


\section{Figure legends}

\section{Figure 1. NLRP3 inflammasome activation by monosodium urate crystals}

NLRP3 must be primed before activation. Priming (Signal 1) is mediated by an NF B-activating pathways, such as a member of the Toll-like receptors (TLRs). This signaling cascade induces the expression of functional inflammasome components such as NLRP3. Monosdium urate crystals provide signal 2 that triggers the assembly of the inflammasome. The interaction of crystals with the plasma membrane promotes a cellular response that is still poorly understood but includes hallmarks of NLRP3 activation including potassium efflux through ion channels, and mitochondrial perturbations leading to the production and release of mitochondrial ROS into the cytosol. Then NLRP3 activating factors such as NEK7 are engaged promoting NLRP3 oligomerization and inflammasome assembly. The adaptor ASC is recruited to the inflammasome and nucleates into prion-like filaments. Caspase-1 is then recruited by ASC and oligomerizes off the ASC filaments leading to autoproteolytic activation of Caspase-1. Active caspase- 1 then promotes the proteolytic cleavage and maturation of proIL-1 $\beta$ into the biologically active IL-1 $1 \beta$. Caspase- 1 also promotes the cleavage of Gasdermin D (GSDMD) to generate an N-terminal cleavage product that oligiomerizes at the plasma membrane, causing the formation of pyroptotic pores. These pores disrupt the integrity of the cellular plasma membrane, and may contribute to the release of inflammatory mediators including IL-1 $\beta$.

\section{Figure 2. IL-1 signaling links inflammasome activation with the inflammatory cascades}

MSU crystals are detected by innate immune cells such as macrophages, monocytes or neutrophils that respond and produce active IL-1 IL-1 $\beta$ signals through the IL-1R complex, composed of the IL-1 receptor (IL-1R1) and its co-factor (IL-1RAcP), leading to recruitment of the adaptor MyD88. The expression of IL1R1 is widespread, present on leucocytes as well as endothelial and synovial cells. This results in the recruitment of effector proteins such as the IRAKs (mostly IRAK4) and TRAF6. This triggers the IKK complex and lead to the phosphorylation and degradation of the 
inhibitor of $\mathrm{NF \kappa B}, \mathrm{I} \kappa \mathrm{B} \quad$ Activation of $\mathrm{NF} \mathrm{B}$, turns on the transcription of cytokines and neutrophil-recruiting chemokines, that will amplify the response and initiate a complex inflammatory cascade.

Figure 3 Checks and balances of gouty inflammation

Multiple regulatory pathways influence the acute inflammatory response to MSU. The interaction between macrophage and the neutrophil is important in the regulation of the acute inflammatory response. Modulators of NLRP3-inflammasome activation include acetate, omega-3 fatty acid and anti-oxidants can dampen IL-1 $\beta$ release. High concentration of neutrophils will also favor NETosis and the formation of NET aggregates, which contain proteases capable of degrading inflammatory cytokines. Finally, the release of TGF $\beta$ by macrophages also acts as a brake on the inflammatory response.

\section{Figure 4 Therapeutic targets in gouty inflammation}

Pathways leading to IL-1 signaling can be inhibited at many different steps. Example of compounds and drugs that have been proposed to affect the various steps are shown. While strategies that target potassium efflux or mitochondrial ROS are quite unspecific, inhibitors of NLRP3 assembly such as colchicine or inhibitors of IL-1 $\beta$ and IL-1R have already been proven effective in gout.

Table 1: anti-IL-1 drugs

Available IL-1 blockers

\begin{tabular}{|l|l|l|l|l|}
\hline Drug name & $\begin{array}{l}\text { Mode of } \\
\text { action }\end{array}$ & $\begin{array}{l}\text { Terminal half } \\
\text { life }\end{array}$ & $\begin{array}{l}\text { Molecular } \\
\text { weight }\end{array}$ & Administration \\
\hline anakinra & $\begin{array}{l}\text { IL-1 receptor } \\
\text { antagonist }\end{array}$ & $4-6$ hours & $17.3 \mathrm{kDa}$ & Subcutaneous \\
\hline canakinumab & $\begin{array}{l}\text { human anti-IL } \\
1 \beta \\
\text { monoclonal } \\
\text { antibody }\end{array}$ & 26 days & $145 \mathrm{kDa}$ & Subcutaneous \\
\hline
\end{tabular}




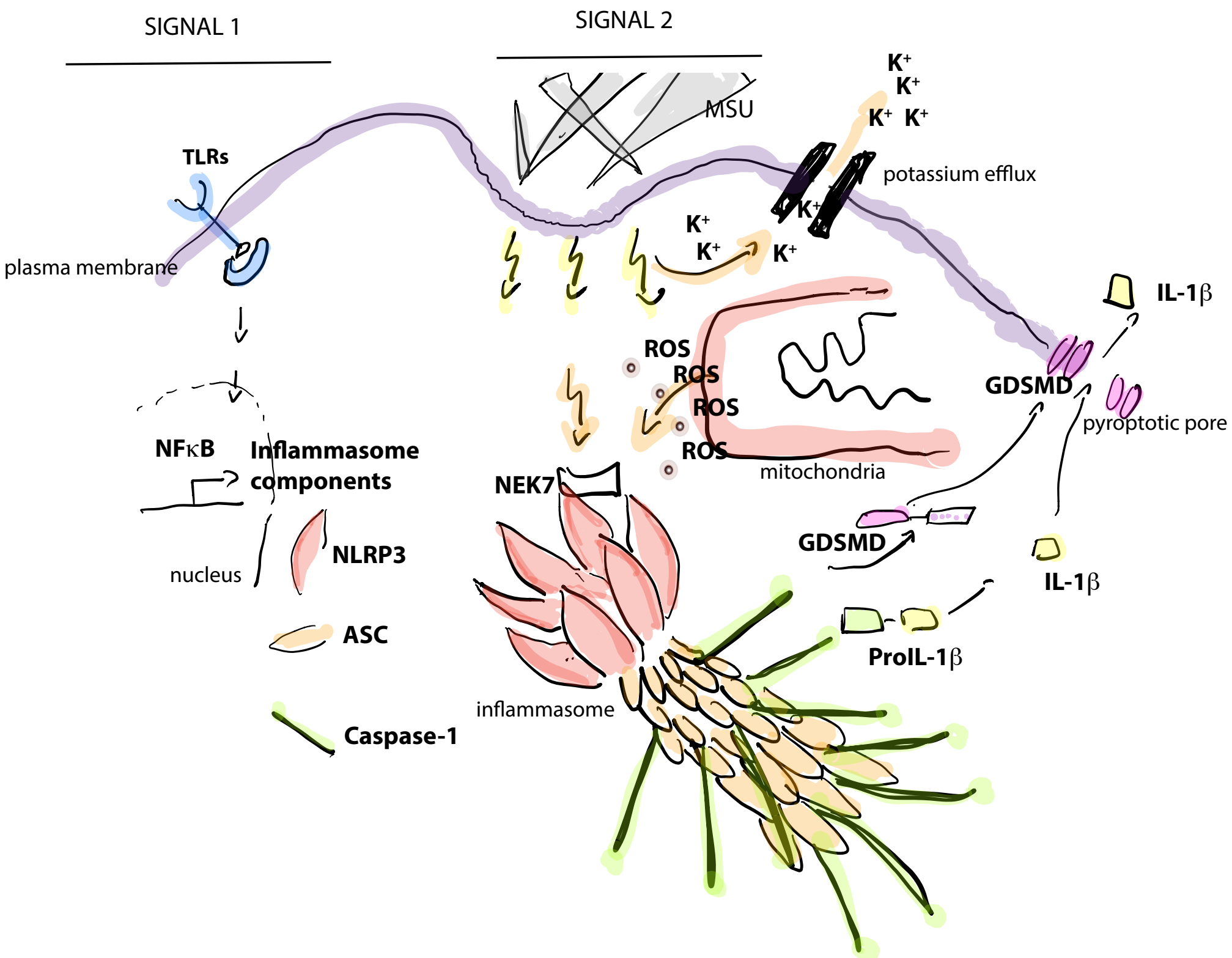




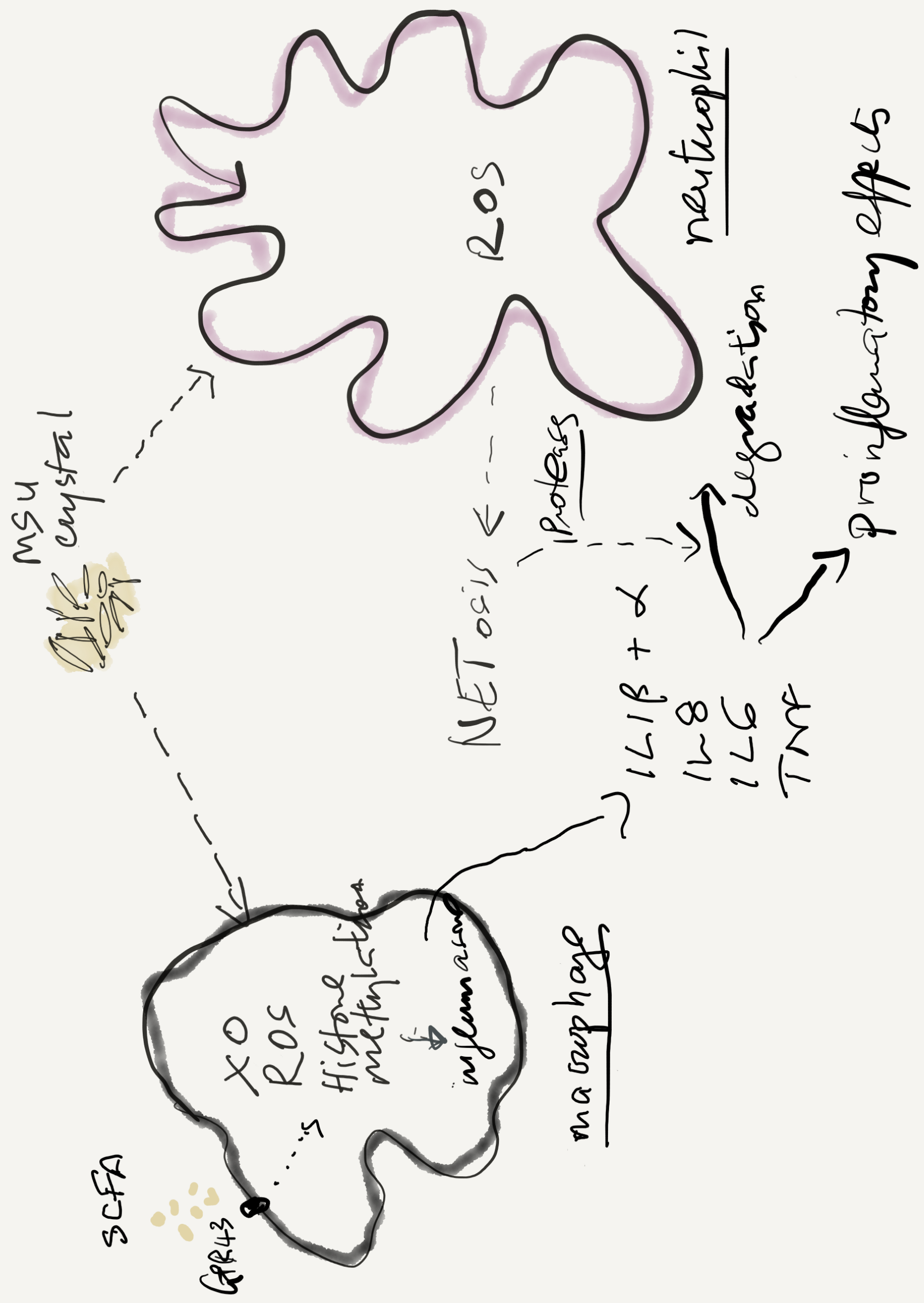


Blockers of potassium efflux

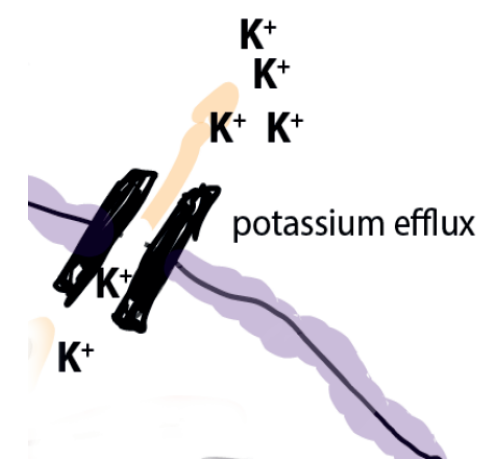

\section{Antioxidants targeting} mitochondrial ROS

Grean Tea molecule:

Epigallocatechin gallate
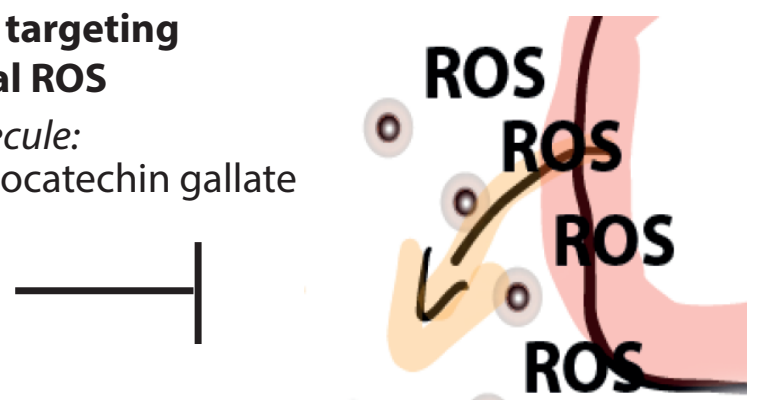

o

\section{NLRP3 inhbitors}

Plant molecule:

Colchicine

Small molecule:

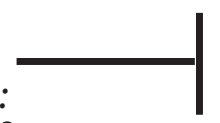

MCC950

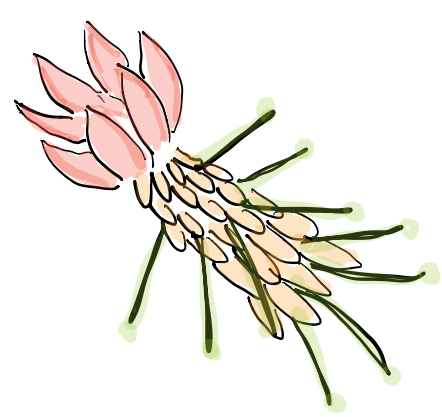

\section{IL-1 $\beta$ processsing inhbitors}

Orally available drug:

VX-765

Caspase-1

Recombinant protein:

AAT-FC<smiles>C1CCC1</smiles>

Serine proteases

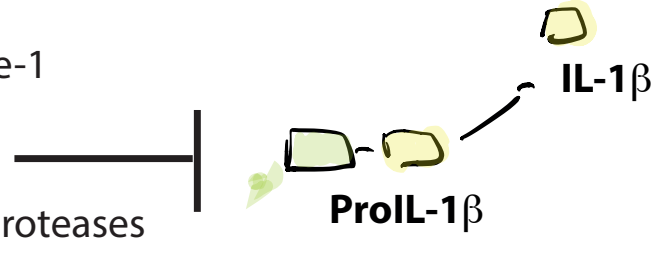

\section{IL-1 $\beta$ inhbitors}

Antibody:

Canakiumab

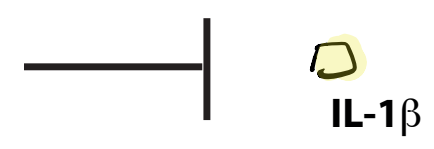

IL-1 receptor inhbitors

Recombinant protein:

Anakinra
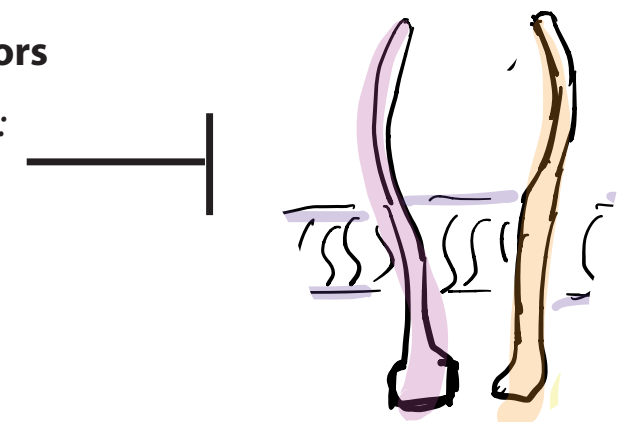